\title{
Analysis of Affect Expressed through the Evolving Language of Online Communication
}

\author{
Alena Neviarouskaya \\ Dept. of Info. and Comm. Eng. \\ University of Tokyo \\ 7-3-1 Hongo, Bunkyo-ku \\ Tokyo 113-8656, Japan \\ Tel: +81-3-5841-6774 \\ E-mail: lena@mi.ci.i.u-tokyo.ac.jp
}

\author{
Helmut Prendinger \\ National Institute of Informatics \\ 2-1-2 Hitotsubashi, Chiyoda-ku \\ Tokyo 101-8430, Japan \\ Tel: +81-3-4212-2650 \\ E-mail: helmut@nii.ac.jp
}

\author{
Mitsuru Ishizuka \\ Dept. of Info. and Comm. Eng. \\ University of Tokyo \\ 7-3-1 Hongo, Bunkyo-ku \\ Tokyo 113-8656, Japan \\ Tel: +81-3-5841-6347 \\ E-mail: ishizuka@i.u-tokyo.ac.jp
}

\begin{abstract}
In this paper, we focus on affect recognition from text in order to facilitate sensitive and expressive communication in computer-mediated environments. Our model for analyzing affect conveyed by text is tailored to handle the style and specifics of informal online conversations. The motivation behind our approach is to improve social interactivity and emotional expressiveness of real-time messaging.
\end{abstract}

In order to estimate affect in text, our model processes symbolic cues, such as emoticons, detects and transforms abbreviations, and employs natural language processing techniques for word/phrase/sentence-level analysis, e.g. by considering relations among words in a sentence. As a result of the analysis, text can be categorized into emotional states and communicative functions. A designed graphical representation of a user (avatar) displays emotions and social behaviour driven by text and performs natural idle movements. The proposed system shows promising results on affect recognition in real examples of online conversation.

ACM Classification: H5.2 [Information interfaces and presentation]: User Interfaces. - Graphical user interfaces (GUI), interaction styles, natural language, theory and methods; I2.7 [Artificial Intelligence]: Natural Language Processing. Language parsing and understanding, text analysis.

General terms: Algorithms, Design, Languages, Theory

Keywords: Affective computing, affective user interface, avatar, emotions, online communication

\section{INTRODUCTION AND MOTIVATION}

"People in virtual communities use words on screens to exchange pleasantries and argue, engage in intellectual discourse, conduct commerce, exchange knowledge, share

Permission to make digital or hard copies of all or part of this work for personal or classroom use is granted without fee provided that copies are not made or distributed for profit or commercial advantage and that copies bear this notice and the full citation on the first page. To copy otherwise, or republish, to post on servers or to redistribute to lists, requires prior specific permission and/or a fee.

IUI'07, January 28-31, 2007, Honolulu, Hawaii, USA.

Copyright 2007 ACM 1-59593-481-2/07/0001...\$5.00. emotional support, make plans, brainstorm, gossip, feud, fall in love, find friends and lose them, play games, flirt, create a little high art and a lot of idle talk. People in virtual communities do just about everything people do in real life, but we leave our bodies behind" (Howard Rheingold [12]).

Social interaction among people is an essential part of every society, and a strong foundation for the development and self-actualization of a person. In everyday life we communicate with each other through multiple informative channels such as natural language, intonation, gaze, facial expressions, gestures and body language. Since people in virtual environments tend to interact in a social way too, the importance of such communication should not be underestimated. Research conducted by Peris et al. [10] argues that "people who use online chats are not only perfectly able to fulfil their social needs in the real world, but they consider online relationships as real as face-to-face relationships".

The necessity to design intelligent user interfaces and to create rich mediating environments for social interactions was a strong incentive for many researchers to analyze natural language with regard to affective information. Original chat or Instant Messaging (IM), e.g. Yahoo! Messenger, which employ animated smiling faces (emoticons) and stylized graphical images (avatars), are recently replacing the purely text based chat systems. The system proposed by Olveres et al. [9] uses a shallow Natural Language Parser for keyword spotting, phrase length measurement and emoticon identification. However, a simple word-level analysis model cannot output an appropriate emotional state in cases where affect is expressed by phrases requiring complex phrase/sentence-level analysis or when a sentence carries affect through underlying meaning. A pure affective keyword spotting technique will fail even with simple sentences like "I climbed the mountain without fear".

More advanced systems for textual affect recognition, such as the Text-to-Emotion Engine [1] or Empathy Buddy [7], perform sentence-level analysis. Both systems use a small set of emotions, the six "basic" types as defined by Ekman [4]. The parser described in [1] generates emotional output only if an emotional word refers to the person him- 
self/herself and the sentence is in present continuous or present perfect continuous tense. We think that such limitations greatly narrow the potential of textual emotion recognition. As the result, sentences like "He is annoying" and "It was the most awful feeling!" are disregarded by the parser despite the fact that they evidently carry affect. An approach aimed at understanding the underlying semantics of language using large-scale real-world commonsense knowledge is proposed by Liu et al. [7], who developed a commonsense affect model enabling the analysis of the affective qualities of text in a robust way.

The weakness of most affect recognition systems integrated with a chat or e-mail browser, however, is that they do not take into account crucial aspects of informal online conversation such as its specific style and evolving language. In order to account for the peculiarity of this medium, and to ensure satisfactory results on real examples, we investigated style, linguistic and interactional features of online conversations, and considered them while constructing our affect analysis model.

The main goal of our research is thus to enrich social interactivity and affective expressiveness of online IM communication. Here, a key issue is to support the automation of multiple expressive channels so that the user does not have to worry about visual self-presentation as in standard IM systems, but can focus on the textual content of the conversation. Our approach is based on deep word/ phrase/sentence-level analysis of affect in text, and the visual reflection of affective states, communicative functions and idle states through use of a 2D cartoon-like avatar.

\section{FEATURES OF INSTANT MESSAGING CONVERSATION}

IM has proven to be one of the most popular online applications. As stated by Shiu et al. [13], younger Internet users employ IM in greater number and more ardently than older generations. Let us see a real example (annotated) of a chat conversation taken from the Yahoo! chat room "Music".

A: sorry if $u$ [you] hear a bad song, just let me know and i'll stop it

B: no problem, hell I am just glad to see a real person for a change, lol [laughing out loud], so many bots

A: I'll take that as a compliment ;) thnx [thanks]

B: $y w$ [you are welcome], lol, kinda to say too much without it soundy corny.

\section{B: damn, I hate when I type too fast and leave out words}

A: its not that, its because ur [your] brain is faster than ur fingers

\section{A: its uncontrollable}

\section{A: at times}

When online, participants do not generally formulate long arguments. These would be considered as a monologue and, therefore, impolite because they bar the others from contributing and upset the conversational mode. IM conversa- tions are typed and often do not contain much, if any, punctuation because communication is informal [3]. As a result, conversations consist of short thoughts and phrases.

Davey et al. [3] state that the lexicon of IM terminology is in a state of constant flux, i.e. evolving. IM users have developed their own language where speed prevails over correct spelling, contributing thus to a greater interactivity. Authors have named this a "shorthand revolution" with the possible drawback of deterioration of today's Standard English. About $76 \%$ of the respondents reported using abbreviations in their IM conversations [3]. One aspect about abbreviations is the motivation behind them: "it is faster and requires less typing". However, since IM participants have different levels of abbreviations in use, they often find it annoying when abbreviations are used without surrounding context to help them understand their meaning [5]. We are thus proposing to resolve the problem of abbreviation understanding in online communication by displaying meaningful transcriptions (e.g. in contrast colour and enclosed in brackets) along with the original text. Output of textual messages in clearly readable form might help new users to avoid confusions, learn and understand IM language. In our research, the problem of correct processing of text by syntactical parser is settled by replacing abbreviations by their transcriptions.

The ability to express emotions in text and self-presentation are very important for a social and friendly atmosphere. Trends show that messaging text is often enriched by symbolic conventions (emoticons, capital letters etc.) to be more expressive. Despite the playful nature of these conventions, the expressions of emotion conveyed are, according to Reid [11], "not in any way thought to be shallow or ephemeral".

\section{AFFECT ANALYSIS MODEL}

In order to identify emoticons, abbreviations, and words, a special database was created using MySQL 5.0 [8]. We collected 364 emoticons, both of American and Japanese style (for example, ":))" and "(^ $\left.\mathrm{O}^{\wedge}\right)$ " for laughing), and the 337 most popular acronyms and abbreviations. As for the affective lexicon, 1620 words were taken from WordNet-Affect [14]. The database contains not only words that refer directly to emotions, mood, traits, attitude etc., but also words (especially, verbs) that carry the potential to provoke affective states in humans. Modifiers, such as "very", "extremely", "not", "less", etc. were collected as well.

As the purpose of affect recognition in an IM system is to relate text to avatar emotional expressions, emotional categories were confined to those that can be visually expressed. For text categorization, we have decided to use nine emotional states taken from a set of ten emotions defined by Izard [6]: 'anger', 'disgust', 'fear', 'guilt', 'interest', 'joy', 'sadness' ('distress'), 'shame', and 'surprise'; and five communicative functions ('greeting', 'thanks', 'posing a question', 'congratulation', and 'farewell').

Emotion categories and intensities were manually assigned to emoticons, some of the abbreviations, and words by three independent annotators. Intensity values range from 0.0 to 1.0 , and describe the intensity degree of affective states from 
'very weak' to 'very strong'. For example, 'cheerful', 'glad', 'happy', 'joyful' and 'elated' all correspond to the 'joy' emotional state, but to a different degree of intensity. In case of emoticons and abbreviations, it is reasonable to relate each entry to only one category. As for the affective words, some of them were assigned to more than one category, because a word can describe a blend of more than one emotion. For instance, two affective states, 'anger' and 'sadness', are involved in the "disappointed" emotion with intensities 0.2 and 0.6 , respectively. The reliability of annotations was measured using Fleiss' Kappa statistics. The calculated Kappa coefficient is 0.94 for emoticons, and 0.93 for abbreviations, showing great labelling reliability. In order to eliminate errors in intensity estimation due to subjective judgements, variance of data from the mean was taken into consideration. If the variance was less than or equal to 0.027 , the resulting intensity was measured as the average of intensities given by three annotators. Otherwise, the intensity value responsible for exceeding the variance threshold was cancelled, and only the remaining values were taken into account. Coefficients for intensity degree strengthening or weakening were given to modifiers (e.g. 1.4 for "very").

The algorithm for analysis of affect in text consists of five stages. In the first stage, we check the sentence for emoticons, abbreviations, interjections, "!" and "?" marks. If there is an emoticon or abbreviation related to an emotional state, no further analysis of affect in text is performed based on the assumption that the emoticon (or abbreviation) dominates the affective meaning of the entire (simple or compound) sentence. It is known that people type emoticons and emotional abbreviations to show actual feeling, or to avoid misleading the other participants, for instance, after irony or joke. In the sentence taken from the real example, "no problem, hell I am just glad to see a real person for a change, lol, so many bots", we consider the abbreviation "lol" (laughing out loud), to be the dominant emotion, which is related to the 'joy' category with intensity 1.0. On the other hand, if there are multiple emoticons or emotion-relevant abbreviations in the sentence, we determine the prevailing (or dominant) emotion based on the following two rules: (1) when emotion categories of the detected emoticons (or abbreviations) are the same, the higher intensity value is taken for this emotion; (2) when they are different (e.g. 'sad': 0.5 and 'joy': 0.2), the category (and intensity) of the emoticon occurring last is dominant. Interjections such as "ouch", "wow" etc. are analysed as well, because these words are added to text to convey emotion.

Regarding the visualisation by the avatar, when both emotional state and communicative function category appear in a sentence, for example, "I'll take that as a compliment ;) thnx", two animations ('joy' with intensity 0.3 and 'thanks') are sequentially displayed. An exception from this rule is the communicative function 'posing a question', which is currently not displayed along with any emotional animation. (Note that while some researchers do not consider questions as emotional at all, we believe that some questions, like "Why do you irritate me?", may carry emotional content.)
In the second stage - before the analysis of the syntactical structure of a sentence - non-categorized abbreviations are replaced by their proper meanings defined in database. Then, the sentence is processed by a deep syntactical parser, Connexor Machinese Syntax [2]. This parser returns a comprehensive description for each word: dictionary form, part of speech, dependency function, syntactic function tags, and morphological tags. All these data are processed further.

In the third stage, word-level analysis is performed. For each word found in the database, either the communicative function category is taken as a feature or the affective features of a word are represented as a vector of emotional state intensities e $=$ [anger, disgust, sadness, fear, guilt, interest, joy, shame, surprise] (e.g. e $=[0.2,0,0.6,0,0,0,0,0,0]$ for word "disappointed"). As the database contains words in their base form, in order to increase intensity of emotional vectors of adjectives in comparative or superlative form, we multiply the vectors by the values 1.2 and 1.4 , respectively.

The fourth stage is devoted to phrase-level analysis. We have defined types of phrases and rules for processing them:

- $\quad$ adjectival phrase ("extremely sad"): modify the vector of adjective;

- noun phrase ("wonderful peace"): output vector with the maximum intensity within each corresponding emotional state in analysing vectors (for instance, $\mathrm{e} 1=[0 . .0 .7 .$.$] and \mathrm{e} 2=[0.3 . .0 .5 .$.$] yield e 3=[0.3 . .0 .7 .]$.$) ;$

- $\quad$ verb plus noun phrase: if verb and noun phrase have opposite valences ("break favourite vase", "enjoy bad weather"), consider vector of verb as dominant; if valences are the same, output vector with maximum intensities in corresponding emotional states for positive ("like honey"), and output null vector for negative;

- $\quad$ verb plus adjective phrase ("is very kind", "feel bad"): output vector of adjective phrase.

The rules for modifiers that influence the emotional vectors of related words are as follows:

- $\quad$ intensifiers multiply or decrease emotional intensity values;

- negation modifiers such as "no" or "not", and connector "neither...nor" cancel (set to zero) vectors of the related words, i.e. "neutralize the emotional content";

- prepositions such as "without", "except", "against", "despite" cancel vectors of related words.

Statements with words like "think", "believe", "sure", "know" and with modal operators such as "can", "may", "need" etc. are not considered by our system because they express a modal attitude towards the proposition. Conditional clause phrases beginning with "if", "when", "whenever", "after", "before" are disregarded as well. 
In the fifth and final stage, the overall affect of a sentence is estimated. We are proposing to differentiate the strength of the resulting emotion depending on the tense of a sentence and availability of first person pronouns. Consideration of tense is very important because "emotions typically occur in response to an event, usually a social event, real, remembered, anticipated, or imagined" [4] (emphasis added by authors). As to first person pronouns, people tend to use them to underline strength of emotion. Many researchers neglect these phenomena. They ignore the difference between "I am annoyed by him" vs. "He is annoying", and some of them completely disregard sentences in past or future tense and without first person pronouns. According to our proposal, the emotional vector of a simple sentence (or of a clause) is multiplied by the corresponding empirically determined coefficient of intensity correction (see Table 1).

\begin{tabular}{|c|c|c|}
\hline \multirow{2}{*}{ Tense } & \multicolumn{2}{|c|}{ First person pronouns } \\
\cline { 2 - 3 } & yes & no \\
\hline present & 1 & 0.8 \\
\hline past & 0.8 & 0.4 \\
\hline future & 0.4 & 0 \\
\hline
\end{tabular}

Table 1: Coefficients of intensity correction.

After the dominant emotion of the sentence is determined, the relevant parameters are sent to the animation engine.

\section{VISUALIZATION OF AFFECT}

We created an emotively expressive avatar for visual reflection of textual affective information. To achieve believable emotion display, the avatar can display various emotions (Figure 1), behaviour associated with communicative functions, and idle states giving a sense of "liveliness". The strength of the displayed emotion is directly related to the intensity of the emotion derived from the text message.

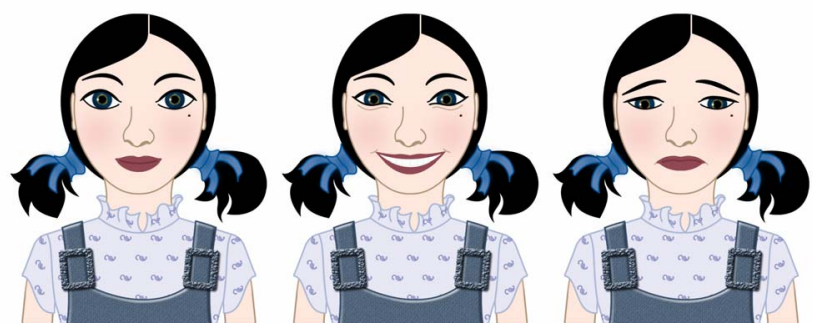

Figure 1: 'Neutral', 'joy' and 'sadness' expressions.

\section{CONCLUSION}

This paper has introduced a novel approach to affect recognition from text. Typically, researchers in this field deal with grammatically and syntactically correct textual input. By contrast, our analysis of affect is inspired by the evolving language as seen in online conversation, for instance, Instant Messaging. The purpose of our work is to improve expressiveness and interactivity of computer-mediated communi- cation. For textual input processing, the proposed analysis model takes into consideration features of IM conversation. Affect in text is classified into nine emotion categories, and information that can be displayed by avatar gestures as communicative behaviour is identified. The strength of a displayed emotional state depends on emotional vectors of words, relations among them, tense of sentence and availability of first person pronouns. An avatar performs various expressive patterns, contributing thus to rich interactivity.

\section{REFERENCES}

1. Boucouvalas, A.C. Real Time Text-to-Emotion Engine for Expressive Internet Communications. Being There: Concepts, effects and measurement of user presence in synthetic environments. Ios Press (2003), 306-318.

2. Connexor Oy. http://www.connexor.com/.

3. Davey, T., Envall, A., Gernerd, M., et al. Instant Messaging: Functions of a New Communicative Tool. Anthropology 427: Doing Things with Words, 2004.

4. Ekman, P. Facial Expression and Emotion. American Psychologist 48, 4 (1993), 384-392.

5. Grinter, R.E., Eldridge, M.A. y do tngrs luv 2 txt msg? In Proc. ECSCW 2001, 219-238.

6. Izard, C.E. Human emotions. NY: Plenum Press, 1977.

7. Liu, H., Lieberman, H., Selker, T. A Model of Textual Affect Sensing using Real-World Knowledge. In Proc. IUI 2003, 125-132.

8. MySQL 5.0. http://www.mysql.com/.

9. Olveres, J., Billinghurst, M., Savage, J., Holden, A. Intelligent, Expressive Avatars. In Proc. of the WECC'98, 1998, 47-55.

10. Peris, R., Gimeno, M.A., Pinazo, D., et al. Online Chat Rooms: Virtual Spaces of Interaction for Socially Oriented People. CyberPsychology \& Behavior 5, 1 (2002), 43-51.

11. Reid, E.M. Electropolis: Communication and Community on Internet Relay Chat. Honours Thesis, University of Melbourne, Australia, 1991.

12. Rheingold, H. The Virtual Community: Homesteading on the Electronic Frontier. Menlo Park, CA: Addison-Wesley Publishing Co., 1993.

13. Shiu, E., Lenhart, A. How Americans Use Instant Messaging. Pew Internet \& American Life Project, Washington, 2004.

14. Strapparava, C., Valitutti, A. WordNet-Affect: an Affective Extension of WordNet. In Proc. LREC 2004, 1083-1086. 